\title{
Preparação para Olimpíada Brasileira de Informática Nível Sênior: Um Relato de Experiência
}

\author{
Paulo Miranda e Silva Sousa, José Robertty de Freitas Costa, \\ Gustavo Ivens Oliveira Silva, Victor de Souza Lima, \\ Wladimir Araújo Tavares, Carla Ilane Moreira Bezerra \\ ${ }^{1}$ Universidade Federal do Ceará (UFC) - Quixadá - CE - Brasil \\ \{paulomirandamss, robertty, gustavo_ivens\}@alu.ufc.br \\ vitinhosouzajuatama@gmail.com, \{carlailane, wladimirtavares\}@ufc.br
}

\begin{abstract}
Programming competitions are playing an important role in developing programming and problem-solving skills. One of the programming competitions aimed at students in the first year of undergraduate, is the Brazilian Computer Olympics (OBI). One of OBI's main challenges is to offer an activity that can acclimatize students and addresses competition subjects. In this context, this paper presents the experience report of the preparation for Senior Level OBI. To support the preparation of OBI, a platform was built, called Code Marathon. In addition, the results of the application and evaluation over three years with IT undergraduate students are presented.
\end{abstract}

Resumo. As competições de programação estão assumindo um papel importante no desenvolvimento das habilidades de programação e resolução de problemas. Uma das competições de programação voltada para os alunos do primeiro ano de graduação, é a Olimpíada Brasileira de Informática (OBI) Nível Sênior. Um dos principais desafios da OBI é oferecer uma atividade que seja capaz de ambientar os alunos e aborde assuntos da competição. Neste contexto, este trabalho apresenta o relato de experiência da preparação para OBI Nível Sênior. Para suportar a preparação da OBI foi construída uma plataforma, denominada Code Marathon. Além disso, são apresentados os resultados da aplicação e avaliação ao longo de três anos com alunos de graduação de TI.

\section{Introdução}

As Diretrizes Curriculares Nacionais para os cursos de graduação em Computação estabelecem que as seguintes habilidades devem ser desenvolvidas ao longo da graduação: ser capaz de reconhecer o valor do pensamento computacional na vida cotidiana; ser capaz de usar o pensamento computacional em circunstâncias apropriadas; e, ser capaz de criar soluções criativas para problemas complexos [BRASIL 2016]. Neste contexto, as competições de programação no Brasil, como Olimpíada Brasileira de Informática (OBI) e a Maratona de Programação, surgiram como uma tentativa de desenvolver o pensamento computacional através da proposição de problemas que estimulam a criatividade.

Gradativamente, as competições como concursos de programação assumiram um papel central na transmissão de certas habilidades que não são transmitidas nas salas de aula. Inovações como concursos de programação competitiva ainda 
são um paradigma emergente na pesquisa educacional, e seus estudos podem contribuir para melhorar as habilidades de resolução de problemas dos estudantes de computação [Raman et al. 2018, Moura et al. 2019]. Essas competições tem o objetivo de semear talentos e auxiliar na melhoria do desempenho acadêmico desses alunos [Vitorino et al. 2018, Solórzano et al. 2019]. Além disso, se desenvolve habilidades de trabalho em equipe, preparando os alunos para entrar no mercado de trabalho [Bloomfield and Sotomayor 2016].

A OBI é uma competição científica promovida pela Sociedade Brasileira de Computação (SBC) e organizado pela Unicamp ${ }^{1}$ [de Oliveira ANIDO and MENDERICO 2007]. O objetivo da OBI é provocar nos alunos o interesse pela computação através da proposição de problemas desafiadores voltado para situações do cotidiano. A OBI é dividida em duas Modalidades: Iniciação (ensino fundamental) e Programação (ensino fundamental, ensino médio e graduação). A Modalidade de Programação é dividida em níveis: Nível Júnior, Nível 1, Nível 2 e Nível Sênior. Particularmente, o Nível Sênior é voltado para os alunos do quarto ano do Ensino Técnico e os que estão pela primeira vez em um curso de graduação. Além disso, destaca-se que a OBI é realizada em 3 fases: Local, Estadual e Nacional.

Embora haja boas evidências de benefícios para a organização de concursos de programação, a participação dos estudantes é amplamente voluntária por natureza e não é obrigatória para a concessão de diplomas acadêmicos por institutos de ensino superior ou para a contratação ou aumento de salários pelas organizações [Raman et al. 2018]. Dessa forma, deve haver diversos incentivos para que haja maior participação dos alunos nessas competições.

O objetivo do trabalho é apresentar a experiência de cursos de graduação em TI, da Universidade Federal do Ceará (UFC) - Campus Quixadá, na preparação e participação na OBI na Modalidade de Programação Nível Sênior ao longo de três anos. É apresentada a metodologia realizada para o treinamento da OBI. Além disso, foi desenvolvida uma ferramenta web com o intuito de apoiar os treinamentos. Por fim, são apresentados os resultados de um questionário aplicado com os alunos para analisar os benefícios do treinamento e participação da OBI relacionado ao desempenho acadêmico destes alunos.

\section{Trabalhos relacionados}

[Ramos et al. 2015] apresentam um projeto de extensão que tem como objetivo ensinar programação para alunos do ensino médio, capacitando-os a participar da Olimpíada Brasileira de Informática. Eles utilizaram para apoiar a ação as linguagens Scratch, Python e $C++$. No relato do presente trabalho, o curso preparatório é ofertado para os alunos do primeiro semestre de cursos da área de TI. Além disso, o treinamento é realizado de maneira simultânea com a disciplina de Fundamentos de Programação, reforçando o conteúdo visto durante a disciplina.

Em [Nowicki et al. 2013], os autores apresentam um curso de programação utilizando ferramentas de aprendizagem à distância voltado para os alunos do Ensino Secundário na Polônia. Neste curso, os alunos eram acompanhados online através de competições de programação semanais. Na preparação do nosso relato, as aulas foram

\footnotetext{
${ }^{1}$ https://olimpiada.ic.unicamp.br/
} 
ministradas utilizando ferramentas de videoconferência; parte do conteúdo encontra-se disponível em cursos gratuitos oferecidos pela plataforma Neps Academy e também foi desenvolvida uma plataforma chamada Code Marathon para disponibilização de outros conteúdos; além de ser realizado o acompanhamento dos alunos em simulados semanais.

Já [de Souza and Lopes 2018], apresentam uma ação em uma escola pública visando o desenvolvimento do raciocínio lógico e pensamento computacional. Nesta ação, os autores desenvolveram um conjunto de 5 atividades práticas inspirados nos problemas da OBI da modalidade Iniciação. As avaliações realizadas mostraram que a utilização das dinâmicas aumentaram consideravelmente a quantidade de acertos. Em comum com o nosso relato, a preparação utiliza os problemas de programação como ferramenta para obter o conhecimento teórico e as habilidades para resolução de novos problemas.

Além disso, também existem iniciativas de ferramentas para auxiliar nos treinos para OBI. [Solórzano et al. 2019] apresentam o Pratique OBI, um sistema web que permite a criação de provas personalizadas com questões da OBI na modalidade Iniciação, para serem utilizadas como simulados em treinos para a competição. [Moura et al. 2019] desenvolveram uma aplicação Android com minijogos utilizando conceitos de virtualização aplicados a questões da OBI voltados para alunos de ensino médio e fundamental. Neste trabalho, também desenvolvemos uma ferramenta para apoiar o treinamento da OBI e de maratona de programação, denominada Code Marathon. O diferencial da nossa plataforma é que ela também apoia o treinamento do nível Sênior.

\section{Metodologia}

O objetivo deste trabalho é apresentar o relato de experiência para preparação de alunos de graduações de TI da UFC - Campus Quixadá para competições de programação, em específico a OBI Nível Sênior. Para isso, foi elaborada uma metodologia de apoio ao treinamento e também uma ferramenta web, denominada Code Marathon. O desafio é nivelar vários níveis de conhecimentos e ministrar conteúdos avançados em uma janela de tempo que tem uma duração média de 4 a 6 meses. A metodologia foi divida em quatro etapas, descritas a seguir:

Divulgação do Treinamento para OBI: no início do semestre, os alunos envolvidos no projeto fazem a divulgação da competição nas salas de aulas, explicam a importância da competição para a formação acadêmica e profissional, e informam as datas e horários da realização do curso preparatório para a competição. As aulas começam, geralmente, após um mês do início do semestre letivo, pois neste período os alunos já começam a desenvolver o pensamento lógico para solucionar problemas e terem contato com uma linguagem de programação na disciplina Fundamentos de Programação.

Seleção da Linguagem: durante a disciplina de Fundamentos de Programação, os alunos costumam trabalhar com a linguagem $\mathrm{C}$ ou $\mathrm{C}++$. No curso preparatório avaliado neste trabalho, foi adotada a linguagem $\mathrm{C}++$, pois esta linguagem oferece um grande número de estruturas de dados prontas que auxiliam o processo de desenvolvimento de códigos e também possui grande similaridade com a linguagem C. Além disso, a plataforma utilizada neste trabalho adota a linguagem $\mathrm{C}++$ para trabalhar com assuntos que são abordados na OBI.

Planejamento da Ementa: o curso preparatório tenta abordar a maior quantidade de assuntos significativos da ementa da Modalidade Programação Nível Sênior. Em 
2018, um estudo sobre a ementa foi realizado, verificando que era viável ministrar todo o conteúdo planejado no período de tempo determinado, ou seja, entre o início do período letivo e a data da Fase Nacional da competição. Os critérios utilizados para seleção dos conteúdos foram: dificuldade, pré-requisitos e recorrência em edições anteriores. A ementa abordada evoluiu ao longo dos anos. Na Tabela 1 é apresentada a ementa final planejada para o ano de 2020.

Tabela 1. Ementa do Grupo Preparatório da OBI Nível Sênior em 2020.

\begin{tabular}{|l|l|}
\hline Aula & Conteúdo \\
\hline Aula 1 & Introdução; Entrada e Saída; Principais Erros; Seleção. \\
\hline Aula 2 & Caracteres; Repetição (While e For). \\
\hline Aula 3 & Vetores; Matrizes. \\
\hline Aula 4 & Strings; Funções; Funções Recursivas. \\
\hline Aula 5 & Complexidade; Vector; Pair; Struct; sort) do C++. \\
\hline Aula 6 & Divisibilidade; Algoritmo de Euclides; Primos; Crivo. \\
\hline Aula 7 & Pilha; Fila; Set; Map. \\
\hline Aula 8 & Variações de inteiros; Operações com Bits; Matemática básica; Geometria Computacional. \\
\hline Aula 9 & Busca Completa; Guloso; Divisão e Conquista; Merge Sort; Contagem de Inversão. \\
\hline Aula 10 & Programação Dinâmica part.1 (Introdução, Mochila, LCS, Kadane.) \\
\hline Aula 11 & Programação Dinâmica part.2 (LIS, Troco, Não clássicas.) \\
\hline Aula 12 & Representação de Grafos; DFS; BFS. \\
\hline Aula 13 & Fila de prioridade; Menor caminho; Prim; Floyd-Warshall. \\
\hline Aula 14 & $\begin{array}{l}\text { Árvore Binária de Busca; Árvore de Segmentos; Árvore de Indexação Binária(BIT) e Árvore de Indexação } \\
\text { Binária 2D (BIT2D). }\end{array}$ \\
\hline Aula 15 & LCA; Caminho Euleriano; Grafos Bipartidos. \\
\hline Aula 16 & Union Find; Kruskal; Ordenação Topológica; Bellman-Ford. \\
\hline Aula 17 & Two-pointer; Programação Dinâmica com Bitmask; Caixeiro Viajante. \\
\hline Aula 18 & $\begin{array}{l}\text { Permutações e Combinações; Princípios Combinatórios; Exponenciação de Matrizes para resolver proble- } \\
\text { mas de grafos e de programação dinâmica. }\end{array}$ \\
\hline Aula 19 & Line Sweep; Convex Hull; Compressão de Coordenadas; Teoria dos Jogos. \\
\hline
\end{tabular}

Escolha da Plataforma: como a competição de programação demanda bastante prática, buscou-se uma plataforma que oferecesse os problemas separados por assuntos. Em 2018, foi utilizada a plataforma CodCad, que possibilitou revisar os assuntos abordados e enviar os códigos desenvolvidos para serem testados pelos juízes de forma automática. Em 2019, a plataforma CodCad foi descontinuada e deu origem ao Neps Academy $^{2}$. Em 2019 e 2020, foi feita a migração para a plataforma Neps Academy, que possibilita a simulação de competições.

A plataforma Neps Academy, fornece cursos gratuitos que visam o estudo de conteúdos relacionados a programação competitiva, e apresentam uma boa interseção com os conteúdos abordados neste curso. Todos os códigos desenvolvidos em aula e todas as soluções dos simulados realizados são disponibilizado para os alunos pela plataforma GitHub ${ }^{34}$. Isso possibilita aos alunos a revisão dos códigos desenvolvidos durante o encontro e o estudo das soluções de questões que não conseguiram resolver durante o simulado. As aulas remotas realizadas em 2020, foram gravadas e disponibilizadas via pasta compartilhada no Google Drive . $^{5}$

\footnotetext{
${ }^{2}$ https://neps.academy/dashboard

${ }^{3}$ https://github.com/PauloMiranda98/Grupo-Preparatorio-para-OBI-2019

${ }^{4}$ https://github.com/RoberttyFreitas/Grupo-OBI-2020

${ }^{5} \mathrm{https}$ ://www.youtube.com/playlist?list=PL7sdfFUzrD1AJGGrA0Mn9vOusg81XtBx3
} 


\subsection{Code Marathon}

Code Marathon ${ }^{6}$ é uma plataforma que foi desenvolvida para disseminar os conhecimentos em algoritmos clássicos da Ciência da Computação, podendo ser uma referência para estudantes e para competidores da OBI, Maratona de Programação e outros torneios semelhantes. O conteúdo disponibilizado na plataforma é dividido em 8 tópicos: introdução, matemática, estrutura de dados, técnicas de programação, processamento de string, geometria computacional, algoritmos em grafos e variados. Em cada tópico, existe uma subdivisão no qual um assunto específico é explicado (ver Figura 1).

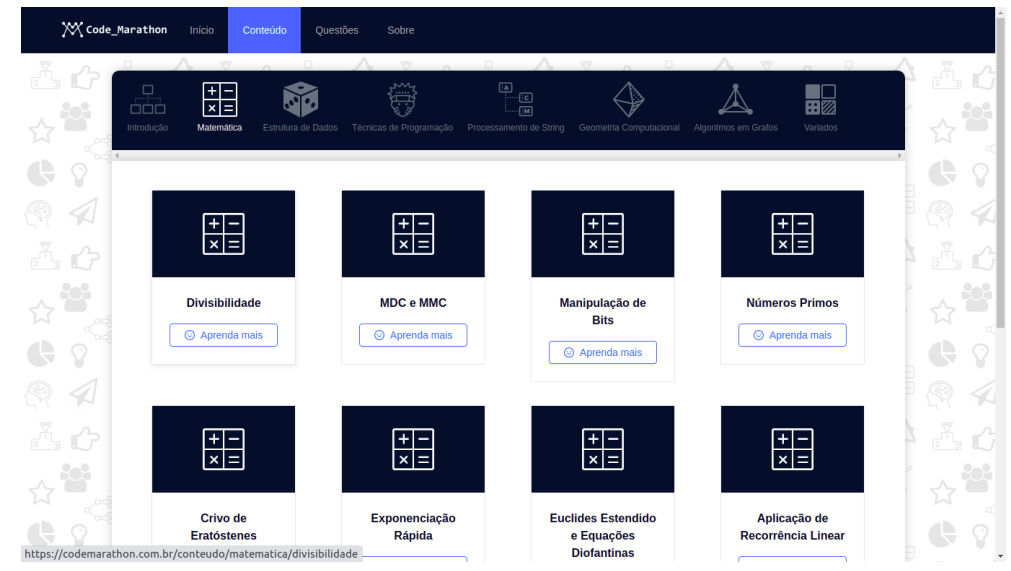

Figura 1. Assuntos do Code Marathon.

A plataforma suporta a escrita de código em $C++$, Java, Python e JavaScript. Além disso, é possível escrever fórmulas matemáticas utilizando a linguagem LaTex e organizar o texto utilizando Markdown, melhorando assim a qualidade e estrutura do texto. Para complementar o texto, a plataforma tem suporte para inclusão de vídeo-aulas diretamente na página (ver Figura 2).

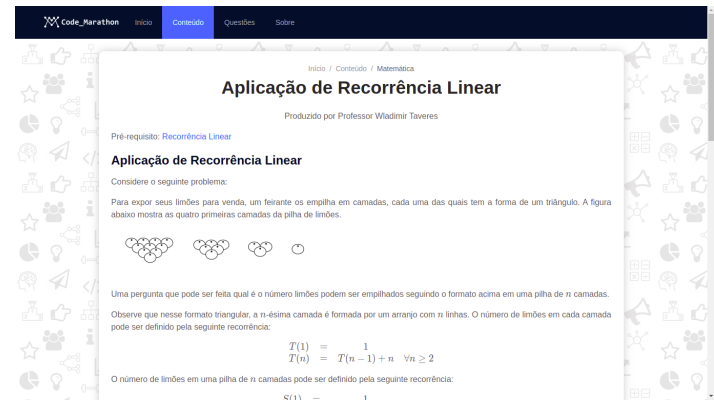

(a) Texto usando LaTex no Code Marathon

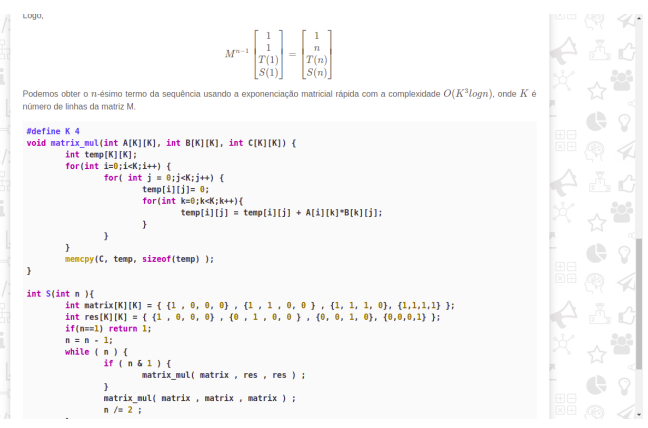

(b) Texto usando C++ no Code Marathon

Figura 2. Plataforma Code Marathon escrita.

Além da parte textual, a plataforma possui um repositório com questões separadas por assuntos. O aluno pode selecionar um tema que acabou que estudar e treinar a partir de várias plataformas disponíveis. Também é possível filtrar por meio da composição de vários assuntos, podendo treinar uma questão que aborda mais de um tema ao mesmo tempo (ver Figura 3).

\footnotetext{
${ }^{6}$ https://codemarathon.com.br/
} 


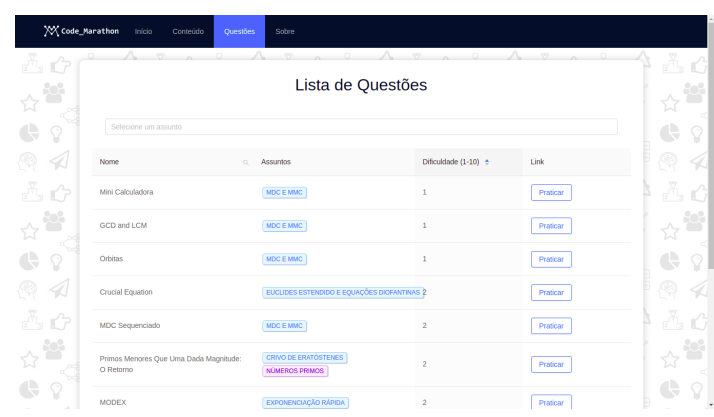

(a) Questões no Code Marathon

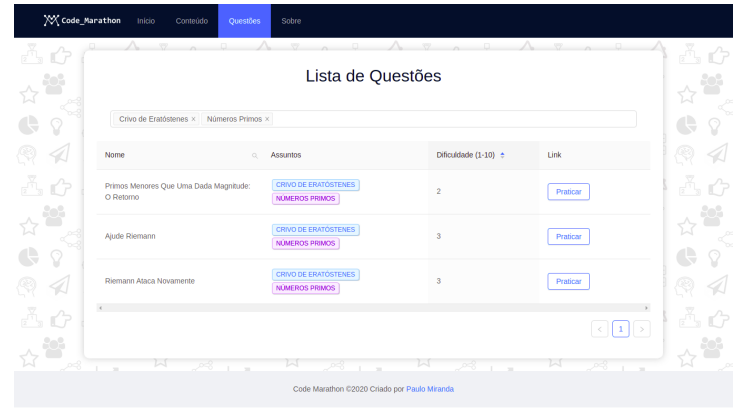

(b) Questões usando filtro no Code Marathon

Figura 3. Plataforma Code Marathon com questões.

\section{Aplicação e avaliação}

A metodologia apresentada para o curso preparatório para OBI nível Sênior foi aplicada em três turmas nos cursos de graduação em TI do Campus da UFC em Quixadá, durante os anos de 2018, 2019 e 2020. As aulas eram semanais com duração de 2 horas. Em 2018 e 2019 as aulas foram no formato presencial. No ano de 2020, a metodologia teve que ser adaptada por causa da pandemia COVID-19. Os encontros passaram a ser realizados de forma remota, utilizando a ferramenta de videoconferência Google Meet. O fato do curso preparatório ser realizado de forma remota permitiu com que alunos de outros Campus também conseguisse acompanhar.

Assim, para analisar a metodologia de treinamento descrita neste trabalho foi disponibilizado aos alunos que participaram do curso em alguma de suas edições, um questionário online ${ }^{7}$ abordando cinco pontos principais: conhecimento prévio em programação e sobre a OBI, engajamento dos participantes, dificuldades encontradas, contribuições trazidas e sugestões de melhoria. A Tabela 2 apresenta o número de participantes das edições e o número de alunos que participaram do curso e responderam ao questionário. A participação no questionário foi voluntária e por isso, não tem-se uma amostra representativa de toda turma.

Tabela 2. Avaliação das turmas para o curso preparatório da OBI Nível Sênior

\begin{tabular}{lcc}
\hline Turmas & Número de participantes do curso & Número de participantes do questionário \\
\hline \hline 2018 & 60 & 13 \\
\hline 2019 & 54 & 13 \\
\hline 2020 & 56 & 19 \\
\hline
\end{tabular}

\subsection{Conhecimento prévio em programação e sobre a OBI}

O primeiro ponto abordado no questionário foi sobre a experiência dos participantes com programação e com a OBI. Foi perguntado aos participantes se os mesmos possuíam algum conhecimento em programação antes de ingressar na universidade, bem como se já conheciam a OBI. 55,5\% dos alunos afirmaram já ter conhecimentos em programação e apenas $42,2 \%$ disseram já conhecer a OBI. Foi perguntado também se já haviam participado da OBI durante o ensino médio e verificou-se que somente 31,1\% afirmaram já ter competido. Percebe-se com as respostas que o pensamento computacional e a OBI parecem ser ainda pouco difundidos entre os alunos do ensino médio.

\footnotetext{
${ }^{7}$ bit.ly/avaliacaoobi20182019 e bit.ly/avaliacaoobi2020
} 


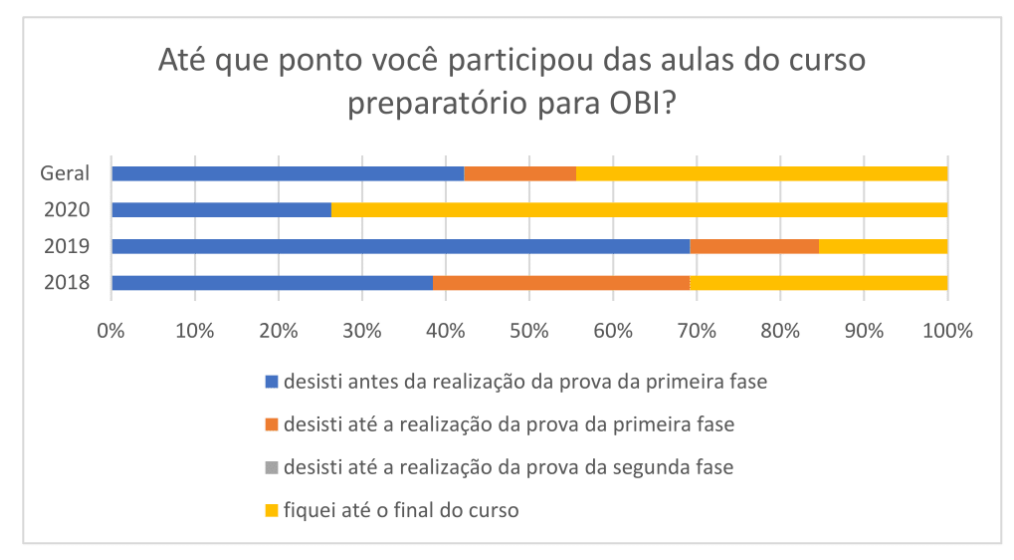

Figura 4. Engajamento dos participantes.

\subsection{Engajamento dos participantes}

Foram destinadas duas perguntas do questionário para avaliar o engajamento dos participantes com o curso preparatório. A primeira delas foi:"Até que ponto você participou das aulas do curso preparatório para OBI?". As respostas para essa primeira pergunta estão ilustradas na Figura 4. Pode-se perceber que 42,2\% dos participantes desistem antes da realização da prova da primeira fase, $13,3 \%$ desistem até a realização da prova da primeira fase e 44,4\% ficam até o final do curso. A segunda pergunta foi: "Até o momento que você participou, qual foi o seu nível de engajamento com o curso?" e vimos que $11,1 \%$ tiveram engajamento ótimo (participar de todas as aulas), 37,8\% tiveram engajamento bom (participar da maioria das aulas), 20\% tiveram engajamento mediano (participar da metade das aulas), 22,2\% tiveram engajamento ruim (menos da metade das aulas) e 8,9\% tiveram engajamento péssimo (participar apenas de uma aula). Observa-se que, embora os participantes gostem das aulas do curso preparatório, existem fatores que fazem com que a maioria não continue participando do curso até o final.

\subsection{Dificuldades encontradas}

No que tange às dificuldades encontradas, foram apresentadas duas perguntas aos participantes. A primeira delas: "Em quais conteúdos você teve maior dificuldade?". A Figura 5 ilustra os resultados desta pergunta, nela é possível perceber que o conteúdo cujos alunos tiveram que empenhar maior esforço foi o de Programação Dinâmica, Geometria Computacional, Complexidade, Grafos, Funções e Funções Recursivas. A segunda pergunta foi: "Quais motivos lhe trouxeram dificuldade durante sua participação no curso?". Dentre os motivos elencados, os principais foram: horário dos encontros $(31,1 \%)$, falta de tempo $(28,9 \%)$, dificuldade em conciliar o treinamento com as disciplinas curriculares $(28,9 \%)$ e dificuldade em acompanhar o ritmo da turma (26,7\%).

Em relação a turma de 2020, cujas aulas aconteceram remotamente devido à pandemia COVID-19, os estudantes que participaram da pesquisa apontaram como principais fatores limitantes: a internet de baixa qualidade, o fato de começarem a trabalhar para ajudar nas despesas de casa e a dificuldade em acompanhar as aulas. A dificuldade de acompanhar as aulas, os alunos justificaram que foi devido: ao conflito de horários com outras aulas, a rotina mais atribulada com as atividades domésticas e a dificuldade de concentração. 


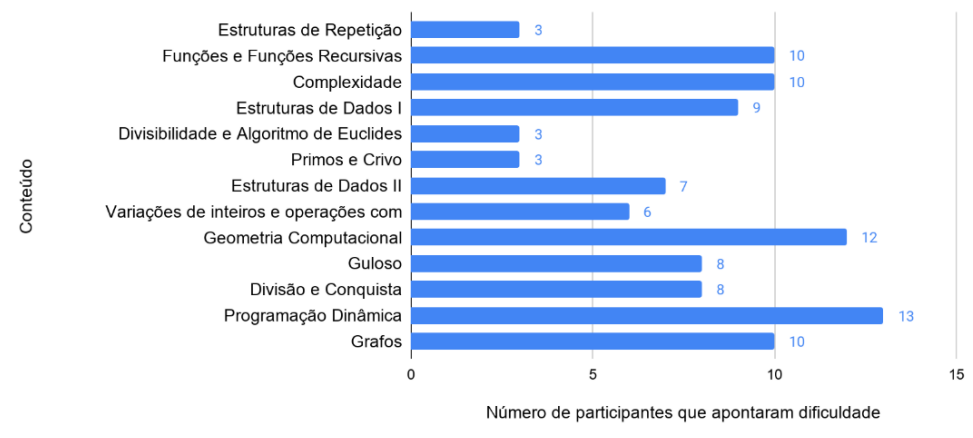

Figura 5. Conteúdos com mais dificuldade.

\subsection{Contribuições}

O feedback dos participantes quanto às contribuições do curso mostrou-se bastante positivo. Ao perguntarmos se gostaram de participar das aulas preparatórias para OBI, quase todos os participantes $(97,8 \%)$ responderam ter gostado de participar. Algo semelhante ocorreu quando foi questionado se o curso contribuiu de alguma forma para a vida acadêmica do estudante: 95,5\% afirmaram que sim. Também foi perguntado se após ter participado das aulas, o aluno sentia-se confiante para realizar as provas da OBI: 44,4\% responderam que sim, $40 \%$ talvez e 15,6\% que não. Foi indagado também se após a participação nas aulas, o aluno sentiu interesse em continuar os estudos em programação competitiva: $66,67 \%$ afirmaram que sim, $20 \%$ talvez e $13,33 \%$ que não.

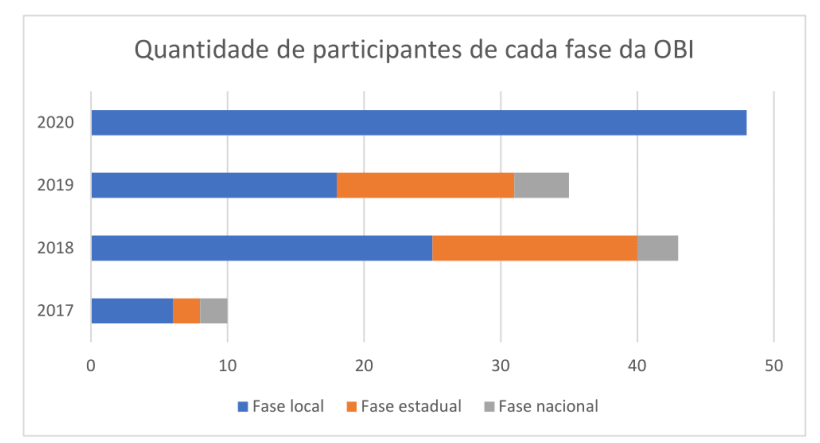

Figura 6. Resultados na OBI.

É importante destacar ainda o crescimento do número de participantes da UFC Campus Quixadá na OBI, sobretudo nas fases iniciais. Pela Figura 6 é possível perceber que antes da implantação do curso a participação da universidade era discreta, com apenas 6 estudantes na fase local, 2 na fase estadual e 2 na fase nacional. Em 2018, primeiro do ano curso, já observa-se um aumento significativo, ocorrendo a participação de 25 alunos na fase local, 15 na fase estadual e 3 na fase nacional. Os números de 2019 foram bem parecidos com os de 2018, com 18 participantes na fase local, 13 na fase estadual e 4 na fase nacional. Em 2020 o número de participantes cresceu: 48 alunos participaram da fase local (700\% a mais que 2017), 10 na fase estadual e 5 na fase nacional. Por fim, foi perguntado de que maneira o curso contribuiu para a vida acadêmica do aluno. A Figura 7 sumariza as respostas para esta pergunta. 


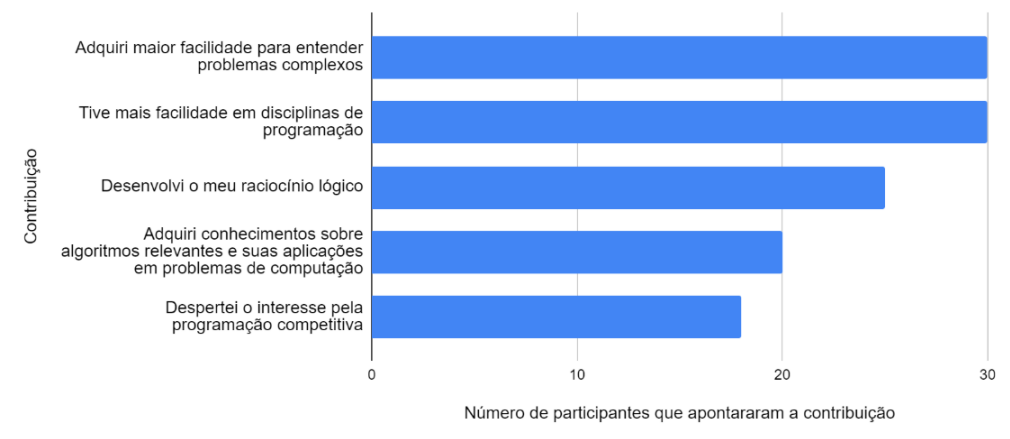

Figura 7. Contribuições trazidas pelo treinamento.

\subsection{Sugestões de melhoria}

Para captar a opinião dos alunos quanto as possíveis adaptações na metodologia, foi realizada a seguinte pergunta através de um campo aberto no formulário: "O que você sugere para melhorar o curso?". Em suma, foi possível perceber que os estudantes gostariam que ocorresse principalmente:

1. A disponibilização de materiais complementares de estudo, como ressaltado pelo participante $\mathrm{P} 34$ em: "Algum conteúdo em forma de PDF ou algo do gênero".

2. A adoção de melhores horários, como sugerem os participantes P24 em "Flexibilização de horários e disponibilização de materiais de estudo" e P22 em "Horário das aulas".

3. Gravação e disponibilização das aulas, como visto nos comentários dos participantes P40 em "Continuar com o método utilizado e gravar todas as aulas" e P45 em "Resolução de mais exercícios, gravar todas as aulas e também gravar as aulas de resoluções de exercícios, abrir um canal no discord para tirar dúvidas no códigos".

Além disso, outras sugestões mostraram-se também bastante interessantes, como a do participante P19 que propõe a realização de mini-maratonas ao final dos encontros em "Maratonas em todo fim de aula... Incentivando a programação competitiva, após encerrado o conteúdo da aula preparatória, poderia ser feito uma mini maratona sobre o conteúdo". Chama atenção também a sugestão dada pelo participante $\mathrm{P} 40$, exposta no item 3 acima, que recomenda a criação de um canal de texto para que haja maior diálogo entre os bolsistas e os participantes do curso.

\section{Conclusão}

Este trabalho apresentou um relato de experiência para preparação dos alunos para OBI nível Sênior. A metodologia contou com apoio da plataforma NepsAcademy e foi desenvolvida a plataforma Code Marathon para armazenamento dos conteúdos abordados na metodologia do curso preparatório. A metodologia desenvolvida foi aplicada em três turmas de cursos de TI do Campus da UFC em Quixadá nos anos de 2018, 2019 e 2020, e foi avaliada a partir de um questionário. Como principais resultados da avaliação teve-se: 0 aumento no número de participantes da instituição em todas as fases da OBI e o despertar do interesse dos estudantes pela cultura da programação competitiva, contribuindo dessa forma para consolidação dessa cultura na universidade. 
Como trabalhos futuros pretende-se: (i) realizar um estudo acerca do desempenho em disciplinas de programação dos alunos que participaram e não participaram do curso, a fim de verificar se ocorre de fato a melhoria do desempenho apontada pela avaliação; e (ii) adaptar a metodologia para que a parte teórica das aulas fique disponível por meio de vídeos e pequenos artigos, visando assim tornar os encontros presenciais mais práticos e dinâmicos através da resolução comentada de exercícios e aplicação de mais simulados.

\section{Referências}

Bloomfield, A. and Sotomayor, B. (2016). A programming contest strategy guide. In Proceedings of the 47th ACM technical symposium on computing science education, pages 609-614.

BRASIL (2016). Ministério da educação. diretrizes curriculares nacionais para os cursos de graduação na área da computação. In RESOLUÇÃO $N^{\circ}$ 5, DE 16 DE NOVEMBRO DE 2016.

de Oliveira ANIDO, R. and MENDERICO, R. M. (2007). Brazilian olympiad in informatics. Olympiads in Informatics, page 5.

de Souza, J. S. and Lopes, A. S. B. (2018). Estimulando o pensamento computacional e o raciocínio lógico no ensino fundamental por meio da obi e computação desplugada. In Brazilian Symposium on Computers in Education (Simpósio Brasileiro de Informática na Educação-SBIE), volume 29, page 1893.

Moura, A. F., Tavares, T., Moreira, J., and Mattos, G. (2019). Virtualização de questões da obi para o desenvolvimento do pensamento computacional. In Anais dos Workshops do Congresso Brasileiro de Informática na Educação, volume 8, page 1334.

Nowicki, M., Matuszak, M., Kwiatkowska, A., Sysło, M., and Bała, P. (2013). Teaching secondary school students programming using distance learning: a case study. In Proceedings of the 10th World Conference on Computers in Education (WCCE 2013).

Raman, R., Vachharajani, H., and Achuthan, K. (2018). Students motivation for adopting programming contests: Innovation-diffusion perspective. Education and Information Technologies, 23(5):1919-1932.

Ramos, T., Batista, L. V., Neto, J. A. M., Santos, A., Machado, K., and Branco, P. (2015). Ensino de programação para olimpíada brasileira de informática. In Anais do Workshop de Informática na Escola, volume 21, page 122.

Solórzano, A., Schneider, C., and Charão, A. (2019). Pratique obi: Um recurso de apoio a treinos para a modalidade iniciação da olimpíada brasileira de informática. In Anais do XXVII Workshop sobre Educação em Computação, pages 453-462. SBC.

Vitorino, M., Silva, H., Sampaio, L., and Gheyi, R. (2018). Perfil dos premiados em olimpíadas de informática e sua influência sobre a educação em computação. In Brazilian Symposium on Computers in Education (Simpósio Brasileiro de Informática na Educação-SBIE), volume 29, page 228. 